\title{
A mono- and intralink filter (mi-filter) to improve false-discovery rates in cross-linking mass spectrometry data
}

\author{
Xingyu Chen ${ }^{1,2 \#}$, Carolin Sailer ${ }^{1,2 \#}$, Kai Michael Kammer ${ }^{1,2}$, Julius Fürsch ${ }^{1,2}$, \\ Markus R. Eisele ${ }^{3}$, Eri Sakata ${ }^{3,4}$ \& Florian Stengel ${ }^{1,2}$
}

${ }^{1}$ University of Konstanz, Department of Biology, Universitätsstrasse 10, 78457 Konstanz, Germany

${ }^{2}$ Konstanz Research School Chemical Biology, University of Konstanz, Universitätsstrasse 10, 78457 Konstanz, Germany

${ }^{3}$ Department of Molecular Structural Biology, Max Planck Institute of Biochemistry, 82152 Martinsried, Germany

${ }^{4}$ Institute for Auditory Neuroscience, University Medical Center Göttingen, 37077 Göttingen, Germany

\# These authors contributed equally to this work

* Correspondence should be addressed to: florian.stengel@uni-konstanz.de

KEYWORDS, mi-filter, false-positive identification, cross-linking mass spectrometry (XL-MS), false-discovery rate (FDR), 


\section{ABSTRACT}

Cross-Linking Mass Spectrometry (XL-MS) has become an indispensable tool for the emerging field of systems structural biology over the recent years. However, the confidence in individual protein-protein interactions (PPIs) depends on the correct assessment of individual inter protein cross-links. This can be challenging, in particularly in samples where relatively few PPIs are detected, as is often the case in complex samples containing low abundant proteins or in in-vivo settings. In this manuscript we are describing a novel mono- and intralink filter (mi-filter) that is applicable to any kind of crosslinking data and workflow. It stipulates that only proteins for which at least one monolink or intra-protein crosslink has been identified within a given dataset are considered for an inter-protein cross-link and therefore participate in a PPI. We show that this simple and intuitive filter has a dramatic effect on different types of crosslinking-data ranging from single protein complexes, over medium-complexity affinity enrichments to proteome-wide cell lysates and significantly lowers the number of false-positive identifications resulting in improved false-discovery rates for inter-protein links in all these types of XL-MS data. 


\section{INTRODUCTION}

An increasingly relevant approach for addressing protein-protein interactions (PPIs) is based on the rapidly evolving technology of crosslinking coupled to mass spectrometry (XL-MS). The general approach of protein XL-MS is based on covalent bonds that are formed using crosslinking reagents between proximal functional groups (most commonly lysine residues) in their native environment ${ }^{1-4}$. The actual crosslinking sites are subsequently identified by mass spectrometry (MS) and reflect the spatial proximity of regions and domains within a given protein (intra-link) or between different proteins (interlink). Additionally, the crosslinker can react twice within one peptide (loop-link) or only on one side with the peptide and hydrolyze on the other side (mono-link), revealing information on the accessibility of a specific amino acid residue. The field has seen significant technological and conceptual progress over the last couple of years and by now various enrichment strategies, different crosslinking chemistries and multiple detection and annotation strategies have been introduced ${ }^{1,2,4}$.

With the structural probing of recombinantly expressed static protein complexes by now being firmly established, it is the recent application of XL-MS on the systems level ${ }^{5}$ and in living cells ${ }^{6}$ that has spurred great interest and an ever-increasing number of studies ranging from bacterial, fungal and mammalian cell lysates and cultured cells ${ }^{7,8}$, specific cellular organelles ${ }^{9-11}$ and tissue ${ }^{12,13}$ have been reported. These studies hint at the exciting prospect that XL-MS will soon be able to facilitate the structural probing of interaction partners of any protein of interest within living cells or even organisms.

However, the confidence in individual protein-protein interactions (PPIs) based on crosslinking data depends on the correct assessment of false discovery rates (FDRs) for individual inter protein cross-links. As recent data shows that FDRs in cross-linking data are frequently underestimated ${ }^{14}$, which is particularly the case for inter-protein cross-links 15 , this can undermine the confidence in individual PPIs and protein networks based on cross-linking data.

To date, FDR assessment in XL-MS has been primarily addressed through the optimization of scoring algorithms and the use of decoy databases 11, 14, 16-18. This approach works particularly well if large enough numbers of identifications can be reached for each particular cross-linking class (i.e. inter-protein, intra-protein and monlink cross-links), but can be challenging in cases where relatively few PPIs are detected, as is often the case in complex samples containing low abundant proteins ${ }^{19}$ or in in-vivo settings. 
In this manuscript we therefore took a different approach and describe a novel mono- and intralink filter (mi-filter) that is applicable to any kind of crosslinking data and analysis pipeline. It stipulates that only proteins for which at least one monolink or intra-protein crosslink has been identified within a given dataset should be considered for an interprotein cross-link and therefore participate in a PPI. We show that this simple and intuitive filter has a dramatic effect on all types of crosslinking-data ranging from single protein complexes, over medium-complexity affinity enrichments to proteome-wide settings and significantly reduces the number of false-positive identifications resulting in improved false-discovery rates in all these types of XL-MS data. 


\section{RESULTS}

\section{Concept of the Mi-filter}

Our "mi-filter" (Monolink/Intralink-filter) is based on the simple idea that only proteins for which at least one monolink or intra-protein crosslink has been identified within a given dataset should participate in an inter-protein cross-link and be part of a legitimate PPI (Figure 1). It is based on the observation that if the abundance of protein is high enough to be detectable by XL-MS, the formation rate of monolinks and intra-protein cross-links will be significantly higher than that of interlinks ${ }^{19}$. Or in other words, if no mono-link or intra-protein cross-link can be detected for a given protein, there is a high likelihood that this protein is not addressable by XL-MS in this particular sample and any inter-protein cross-link that includes this protein is likely a false-positive.

Our approach is therefore not designed as a contradiction to prevalent FDR calculations based on scoring algorithms and the use of decoy databases but is rather intended as an additional tool that can be applied on top of existing workflows in order to minimize falsepositive assignments of inter-protein cross-links or as an alternative, in case no sufficient data coverage for conventional FDR calculations can be achieved.

\section{Inter-protein cross-links are disproportionally affected by high FDRs}

Minimizing false-positive assignments for inter-protein cross-links is particularly crucial as all PPIs based on cross-linking data depend entirely on information from inter-protein crosslinks. Moreover, inter-protein cross-links are disproportionally affected from high FDRs (Figure 2). Figure 2 shows the amount of detected decoy hits for monolinks, intraprotein and inter-protein cross-links for the 26S proteasome at increasingly stringent filtering settings, i.e. increasing agreement between measured experimental and in-silico generated reference spectra. The data shows that the relative proportion of detected decoy hits for inter-protein cross-links is significantly larger than for mono or intra-protein links for all settings and, importantly, that inter-protein cross-links still contain a significant number of false-positive identifications at cut-offs where the number of detected decoys (and thus resulting FDRs) for intra-protein and monlinks are already negligible.

Therefore, in cases where FDRs are assigned in toto over all classes of cross-links or in cases where FDRs based on decoy-measurements cannot be properly calculated, for example due to sparsity of the data, the likelihood of a false-positive assignment for interprotein cross-links increases dramatically. 


\section{Mi-filter improves inter-protein FDRs for different types of cross-linking data.}

In order to evaluate the effect of the mi-filter on FDRs, we applied it to typical cross-linking datasets of different complexity using the $x Q u e s t / X$ Prophet ${ }^{20}$ pipeline as an example (Figure 3). Our least complex sample is the $26 \mathrm{~S}$ proteasome from $S$. cerevisiae consisting of 34 proteins (Figure $\mathbf{3 A}$ and $\mathbf{B}$ ). An intermediate one is the combined dataset of pre-60S ribosomal particles obtained by affinity enrichment, containing a total of around 300 proteins (Figure $\mathbf{3 C}$ and $\mathbf{D}$ ). We could previously show that the application of the mifilter to this dataset results in significantly improved FDRs ${ }^{21}$, but only now we have thoroughly investigated the influence of the mi-filter on this and other datasets and for various settings of increasingly stringent filtering. The most complex sample we evaluated using our mi-filter is a proteome-wide XL-MS dataset of $S$. cerevisiae cell lysate ${ }^{19}$ (Figure 3E and $\mathbf{F}$ ).

We first had a closer look at the relative abundance of proteins that were filtered-out by the mi-filter, taking the pre-60S ribosomal particles dataset as an example ${ }^{21}$. Here proteins for which a mono- or intra-protein link was detected are in average of significantly higher abundance than proteins without mono- or intra-protein links (Supplementary Figure 1). This already indicates that proteins without mono- or intra-protein links are either not present in the sample at a concentration high enough for crosslink identification or they are not present at all.

After application of the mi-filter (right bar of each group) all datasets consistently exhibit a significant decrease in the number of detected decoy inter-protein links (Figure 3A, 3C and $\mathbf{3 E}$ ) as well as a significant decrease in the resulting FDRs (Figure 3B, 3D and 3D). It is interesting to note that this is not only true for the different sample types but also for the increasingly stringent filtering settings (i.e. increasingly good matches between experimental data and and in-silico generated reference spectra). The mi-filter is therefore not only able to filter out most decoy links already at medium filtering settings (Figure 3B, 3D and 3D), it also is able to retain the majority of bona-fide true positive links, as indicated by the diminishing difference of detected target hits between filtered and nonfiltered data for the highest quality MS data (Figure 3A, 3C and $3 E$ ).

Taken together this demonstrates the value of the mi-filter as a stringent filtering device that results in a significant reduction of decoy inter-protein cross-link identifications and improved FDRs for inter-protein cross-links in different types of cross-linking data. 


\section{Quality of the mi-filtered data.}

In a next step we wanted to test and benchmark the mi-filter also for its ability to identify true-positive cross-links in a proteome-wide setting. In contrary to mixtures of purified proteins or protein complexes, which can be benchmarked against existing atomistic highresolution structures in order to obtain true-positive identifications (which differs from the mere calculation of FDRs from decoy-hits), there is no known ground-truth in a proteomewide cross-linking experiment, as the precise protein arrangement within a cell or lysate is unknown.

We therefore took the totality of MS and MS/MS spectra that we had experimentally obtained from a sample of crosslinked 26S proteasome and searched it in a proteomewide setting (i.e. against a large protein database; see methods for details) with and without application of the mi-filter (Figure 4A). Our data shows that the application of the mi-filter did not only lead to a significant reduction of detected decoy-hits and a nearly 35fold reduction in the resulting FDR. When mapped onto the published high-resolution cryo-EM structure of the $S$. cerevisiae $26 S$ proteasome (PDB 4CR2), over $90 \%$ of our mi-filtered cross-links fall within $35 \mathrm{~A}^{\circ}$, the maximal lysine $\mathrm{Ca}-\mathrm{Ca}$ distance that our crosslinker can bridge (Supplementary Figure 2), indicating that our mi-filtered crosslinks are also biologically meaningful.

In summary our data indicates that the mi-filter is not only able to significantly reduce the amount of detected decoy-hits and resulting FDRs but is simultaneously able to identify and retain true-positive cross-links also in a proteome-wide setting. 


\section{DISCUSSION}

In this manuscript we describe and benchmark a novel mono- and intralink filter that is in principle applicable to any kind of crosslinking data and analysis pipeline. This simple and intuitive mi-filter, which removes inter-protein cross-links if the connected polypeptides are not additionally represented within their respective intralink or monolink pools, reduces identification of false-positives and improves FDRs for inter-protein cross-links significantly. We show that this is true for different types of crosslinking-data ranging from single protein complexes, over medium-complexity affinity enrichments to proteome-wide settings. Moreover, in addition to reliably reducing the amount of detected decoy-hits in a given cross-linking sample, the mi-filter is also able to identify and retain the majority of true-positive cross-links, also in a complex proteome-wide setting.

While we have used the $x$ Quest/xProphet cross-linking software suite in this manuscript as an example to benchmark the mi-filter, our mi-filter can in principle be applied to any cross-linking software to further improve and validate the obtained cross-linking data. It can also be used as a stand-alone tool to minimize false-positive assignments and improve the detection of true and biologically relevant PPIs. This is particularly useful in cases where no sufficient data for conventional FDR calculations based on decoys can be achieved, as is often the case in complex samples containing low abundant proteins or in in-vivo settings.

Taken together our mi-filter greatly enhances the reliability of individual inter-protein cross-links in any type of cross-linking data and therefore their ability to provide reliable and biologically relevant positional information as source of a PPI. 


\section{Acknowledgements}

This work was supported by the German Research Foundation through Germany's Excellence Strategy - EXC 2067/1- 390729940, SFB1035/Project A01, and CRC889/Project A11 to E.S. F.S. is grateful for funding from an ASAP Collaborative Research Network Grant (ASAP-000519) and the German Research Foundation (STE 2517/1 and STE 2517/5-1).

\section{Author Contributions}

X.C., C.S. and F.S. conceived the study and experimental approach. M.R.E and E.S. expressed and purified the $26 \mathrm{~S}$ proteasome. X.C. and C.S. implemented and applied the mi-filter with help from K.M.K. J.F. performed original proteome-wide studies. C.S. carried out affinity-enrichments. X.C., C.S., K.M.K and F.S. analysed the data. X.C., C.S. and F.S. wrote the paper with input from all authors.

\section{Declaration of interests}

The authors declare no competing interests. 


\section{Figures and Figure Legends}

\section{Figure 1}
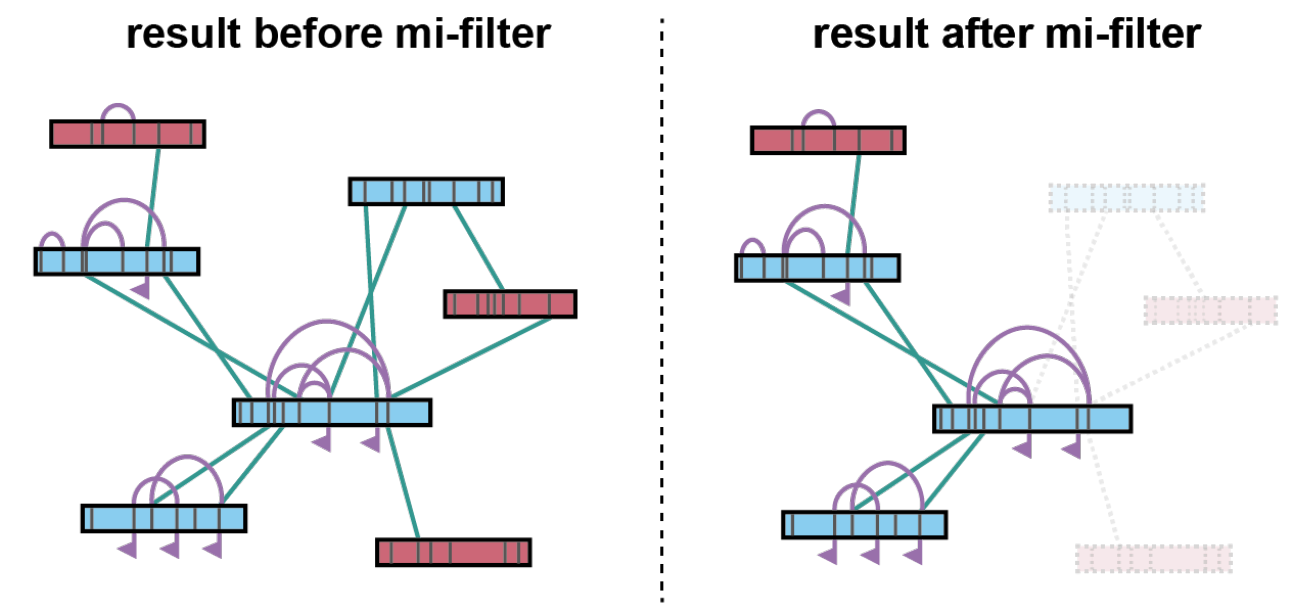

$\square$ hit $\quad \square$ decoy Ilysine $\quad$ monolink $\quad$ intralink /interlink

Figure 1 Concept of the mi-filter. Only proteins that contain at least one identified highconfidence monolink or intra-protein crosslink are considered for inter-proteins cross-links and can therefore be part of a PPI. 


\section{Figure 2}

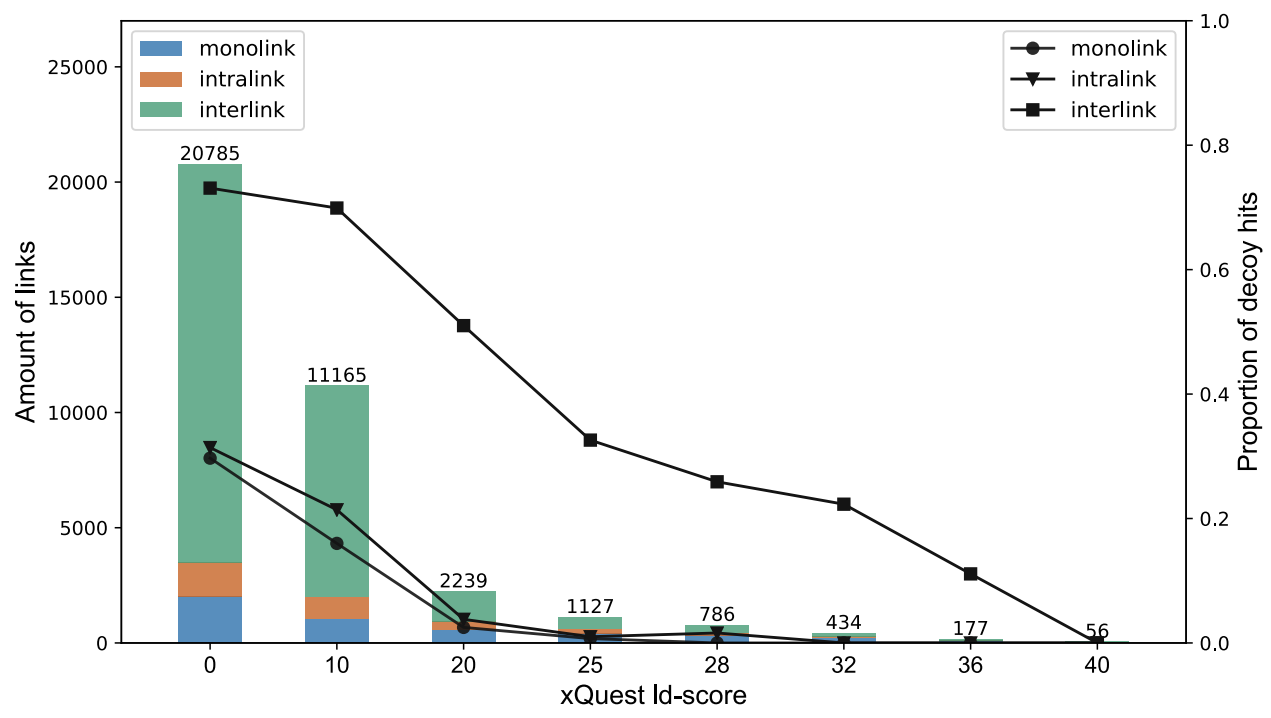

Figure 2 Inter-protein cross-links are disproportionally affected from high FDRs. The bar chart shows the cumulative amount of detected monolinks, intra-protein crosslinks and inter-protein cross-links ( $y$ axis, left) of the $26 \mathrm{~S}$ proteasome at different ld-score cut-offs ${ }^{22}$, i.e. increasing levels of agreement between measured experimental and insilico generated reference spectra. Blotted are the relative proportion of detected decoy hits ( $y$-axis, right) versus the respective ld-score setting ( $x$-axis). 


\section{Figure 3}
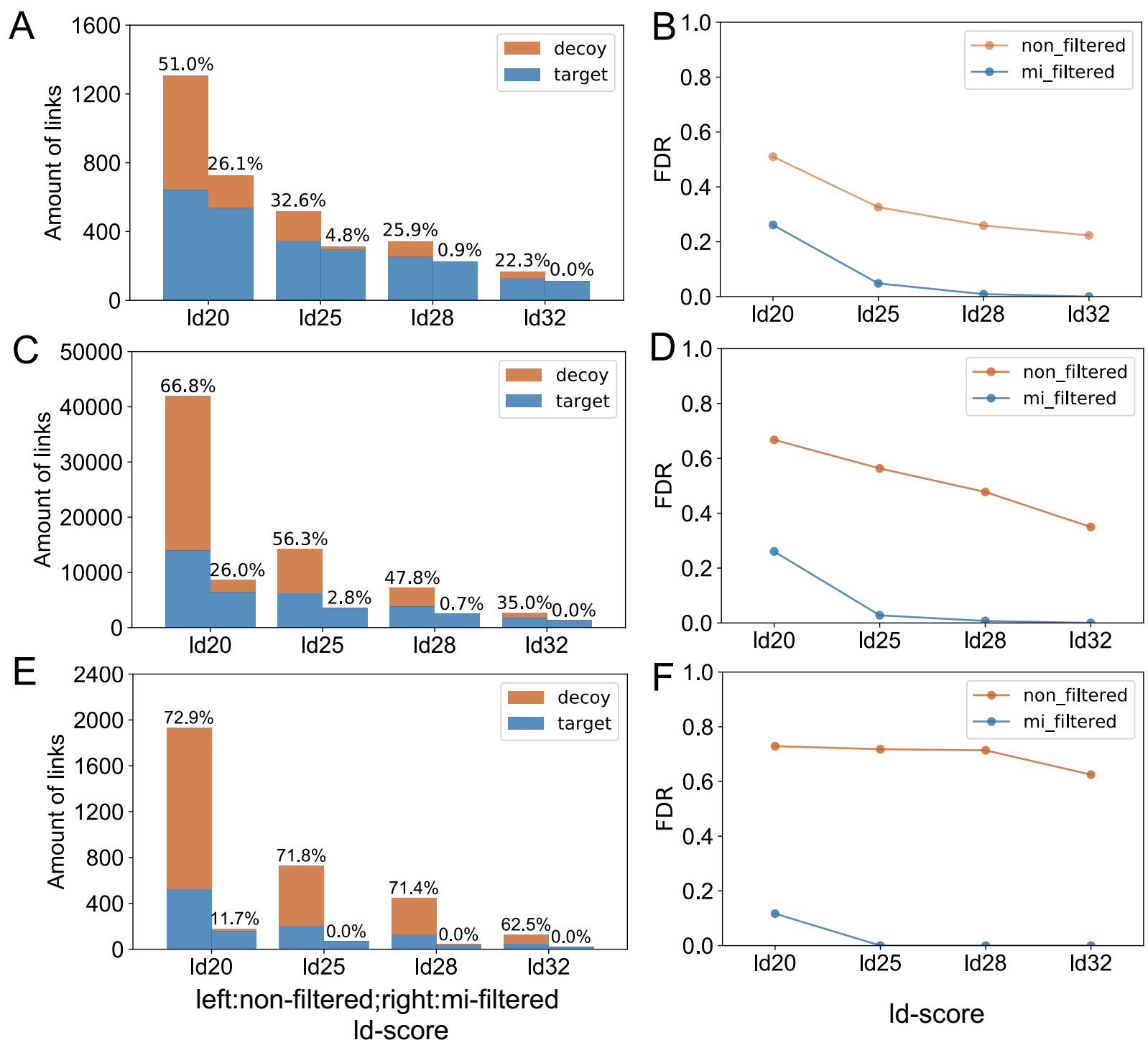

Figure 3 Comparison of FDRs with and without mi-filter. Inter-protein cross-links are shown for three different types of datasets representing typical experimental set-ups including the $26 \mathrm{~S}$ proteasome from $\mathrm{S}$. cerevisiae as an example of a single protein complex (A and $\mathbf{B})$, affinity enrichments of pre-60S ribosomal particles (C and $\mathbf{D})$ and $\mathbf{a}$ proteome-wide cross-linking experiment using $\mathbf{S}$. cerevisiae cell lysate (E and $\mathbf{F})$. Panels A, C and $\mathbf{E}$ show the number of decoy and target hits (non-decoy hits) in non-filtered (left bar in each group) and mi-filtered samples (right bar in each group) for increasingly stringently filtered data. Target hits are shown in blue and decoys in red. The relative percentage of decoy hits for each setting is indicated. Panels $\mathbf{B}, \mathbf{D}$ and $\mathbf{F}$ show the false discovery rate (FDR) of non-filtered (red line) versus mi-filtered results (blue line) for the respective datasets. The FDR was calculated as the proportion of decoy hits to the total amount of all hits (target plus decoy hits). 


\section{Figure 4}

result without mi-filter

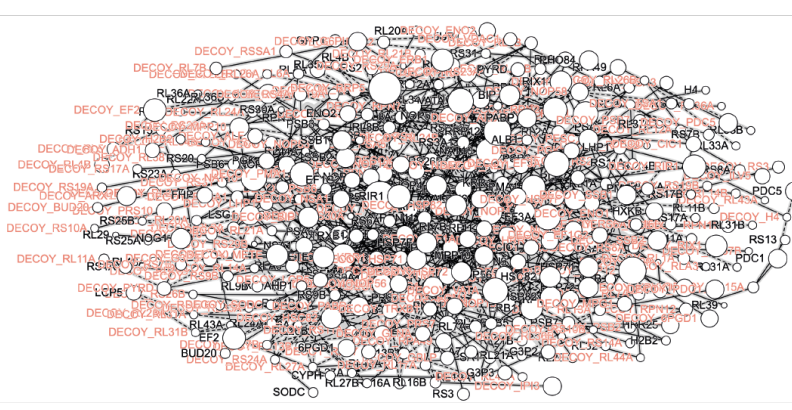

$\mathrm{FDR}=0.36$

\section{result with mi-filter}

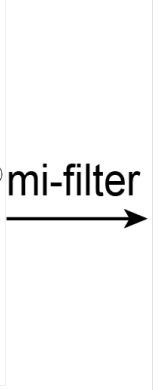

$\stackrel{\text { mi-filter }}{\longrightarrow}$

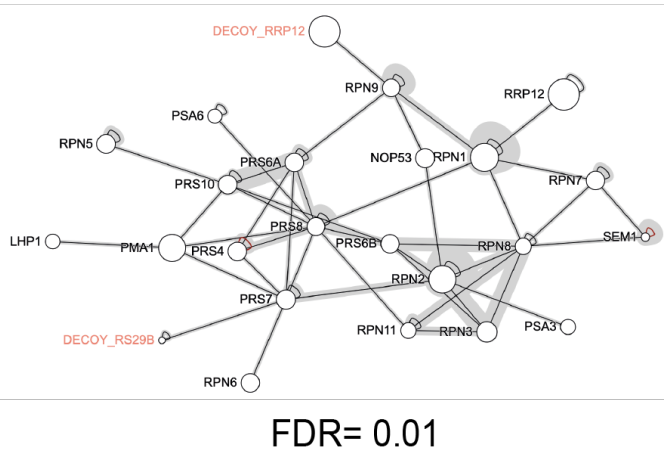

Figure 4 Quality of the mi-filtered data. Shown is a cross-linking dataset of the $26 \mathrm{~S}$ proteasome with and without application of the mi-filter searched against a manually curated database mimicking proteome-wide protein distributions (see methods for details). 


\section{Materials \& Methods \\ Mi-filter script}

The mi-filter script was written in python and is available at the Github repository (https://github.com/xingyu-konstanz/mi-filter.git). It is tailored to $x Q u e s t^{20}$ output tables but can in principle be applied to crosslinking MS datasets obtained by any of the established crosslink-identification software platforms such as MeroX ${ }^{23}, \mathrm{Xlinkx}^{17}, \mathrm{Xi}^{24}$, pLink $2^{25,}{ }^{26}$ or RNPx| ${ }^{27}$. It selects proteins from the input dataset, which contain at least one mono- or intra-protein link and subsequently filters for inter-protein crosslinks within this list. It also calculates a simple FDR (using the ratio of target and decoy links) at each ld-Score cut-off for monolinks, inter-protein and intra-protein cross-links separately.

In detail, the mi-filter script works as follows: it filters the input files for a specified Id-Score, it then concatenates the input data frames and, if specified, filters for biological replicates of cross-linking sites (therefore input files must be sorted by biological replicates). In a next step it adds a 'decoy' column to the concatenated data frame. In the 'XLtype' column, strings are replaced in a way that only three types of cross-link are left: monolinks, intraprotein and inter-protein cross-links. Proteins without a monolink or an intra-protein crosslink are then filtered out by the parameter "-mi "when running the mi-filter program. Subsequently, a simple FDR

$$
\text { FDR = number of decoys } / \text { (number of targets }+ \text { number of decoys) }
$$

is calculated at each Id-Score cut-off (using 1.0 score units) for monolinks, intra-protein and inter-protein cross-links separately. Please note that links containing at least 1 decoy peptide are considered as decoys and there is no discrimination between cross-links of the following type T-T, T-D, D-T and D-D (where T denotes a target peptide and $\mathrm{D}$ a decoy peptide).

\section{5 proteasome cross-linking dataset}

Purification of yeast $26 \mathrm{~S}$ proteasomes was performed as described in ${ }^{28}$. S. cerevisiae cells (YYS40; MATa rpn11::RPN113FLAG-HIS3) were grown for 48 hours and harvested in stationary phase. The purification of 3XFLAG-tagged 26S proteasome was carried out by affinity purification using M2 anti-FLAG beads (Sigma A2220). After incubation for 1.5 hrs at $4^{\circ} \mathrm{C}$ the proteasome was eluted with FLAG peptide. An overnight sucrose gradient was carried out for the second purification step. The sucrose gradient was centrifuged in a Beckman SW41 rotor for $17 \mathrm{~h}$ at $4^{\circ} \mathrm{C}$ at $28000 \mathrm{rpm}$. Proteasome-containing fractions were identified by degradation of the peptide suc- LLVY-AMC, SDS-PAGE analysis, and Bradford assay.

$100 \mu \mathrm{g}$ of purified $26 \mathrm{~S}$ proteasome $(1 \mu \mathrm{g} / \mu \mathrm{l})$ were subsequently incubated with the isotopically labeled crosslinking reagent disuccinimidyl suberate d0/d12 (DSS-H12/D12, Creativemolecules Inc.) at a final concentration of $1 \mathrm{mM}$ for $30 \mathrm{~min}$ at $30^{\circ} \mathrm{C}$ while shaking 
at $650 \mathrm{rpm}$ in a Thermomixer (Eppendorf). The reaction was quenched with ammonium bicarbonate at a final concentration of $50 \mathrm{mM}$ for $10 \mathrm{~min}$ at $30^{\circ} \mathrm{C}$ and $650 \mathrm{rpm}$. Crosslinked samples were dried (Eppendorf, Concentrator plus), resuspended in $100 \mu$ 8M Urea, reduced, alkylated, and digested with trypsin (Promega). Digested peptides were separated from the solution and retained by a solid phase extraction system (SepPak, Waters). Crosslinked peptides were enriched by size exclusion chromatography using an ÄKTAmicro chromatography system (GE Healthcare) equipped with a Superdex ${ }^{\mathrm{TM}}$ Peptide 3.2/30 column (column volume $=2.4 \mathrm{ml}$ ). Fractions were collected in $100 \mu \mathrm{l}$ units and analyzed by LC-MS/MS. For each crosslinked sample two fractions (1.2-1.3 $\mathrm{ml}$ and 1.3-1.4 $\mathrm{ml}$ ) were collected and measured in technical duplicates. Absorption levels at $215 \mathrm{~nm}$ of each fraction were used to normalize peptide amounts prior to LC-MS/MS analysis.

LC-MS/MS analysis was carried out on an Orbitrap Fusion Tribrid mass spectrometer (Thermo Electron, San Jose, CA). Peptides were separated on an EASY-nLC 1200 system (Thermo Scientific) at a flow rate of $300 \mathrm{nl} / \mathrm{min}$ over an 80 min gradient (5\% acetonitrile in $0.1 \%$ formic acid for $4 \mathrm{~min}, 5 \%-35 \%$ acetonitrile in $0.1 \%$ formic acid in $75 \mathrm{~min}, 35 \%$ - $80 \%$ acetonitrile in $1 \mathrm{~min}$ ). Full scan mass spectra were acquired in the Orbitrap at a resolution of 120,000 , a scan range of $400-1500 \mathrm{~m} / \mathrm{z}$, and a maximum injection time of $50 \mathrm{~ms}$. Most intense precursor ions (intensity $\geq 5.0 \mathrm{e} 3$ ) with charge states 3 - 8 and monoisotopic peak determination set to 'peptide' were selected for MS/MS fragmentation by CID at $35 \%$ collision energy in a data dependent mode. The duration for dynamic exclusion was set to $60 \mathrm{~s}$. MS/MS spectra were analyzed in the iontrap at a rapid scan rate.

For the crosslink identification of the 26 proteasome in a "proteome-wide setting" a database was compiled which contained the 34 proteins of the $26 \mathrm{~S}$ proteasome plus the 200 most abundant proteins in $S$. cerevisiae as annotated in the PAX database (https://pax-db.org/). MS raw files were subsequently converted to centroid files and searched using $x$ Quest in ion-tag mode. Crosslinks were exported as .tsv files with the filter settings deltaS $=95$ and a max. ppm range from -5 to 5 , containing all (non-unique) identifications. The mi-filter was applied to different ld-Score cut-offs (20, 25, 28 and 32) and FDRs were calculated as described above and compared to the dataset before mifiltering (Supplementary Data 1 and Figure 3A and 3B).

\section{Pre-60S ribosome XL-MS dataset}

The dataset consists of biological triplicate measurements of 12 different pre-60S ribosomal particles, which were enriched using affinity-tagged RBFs and was collected as part of another study ${ }^{21}$. The mi-filter was applied to different Id-Score cut-offs (20, 25, 28 and 32) and FDRs were calculated as described above and compared to the dataset before mi-filtering (Supplementary Data 2 and Figure 3C and 3D). 


\section{Proteome wide cross-linking dataset}

The dataset contains biological triplicate measurements of cell lysate in Saccharomyces cerevisiae and was collected as part of another study ${ }^{19}$. $x$ Quest results from this paper were directly downloaded and the cross-linked sample using equimolar concentrations (1x) of BS3 as a cross-linker was chosen for further analysis. The mi-filter was applied to different Id-Score cut-offs (20, 25, 28 and 32) and FDRs were calculated as described above and compared to the dataset before mi-filtering (Supplementary Data 3 and Figure 3E and 3F).

\section{Mapping of filtered crosslinks}

Crosslink networks were visualized with xiNET ${ }^{29}$.

\section{Data availability}

The MS raw files, the crosslink databases and original $x$ Quest result files have all been deposited to the ProteomeXchange Consortium via the PRIDE partner repository ${ }^{30}$ with the project accession number PXD031215 (Username: reviewer_pxd031215@ebi.ac.uk Password: yLjErDmt). The previously published ribosome ${ }^{21}$ and lysate ${ }^{19}$ datasets have the project accession numbers PXD021831 and PXD014759, respectively. 


\section{References}

1. Piotrowski, C.; Sinz, A., Structural Investigation of Proteins and Protein Complexes by Chemical Cross-Linking/Mass Spectrometry. Adv Exp Med Biol 2018, 1105, 101-121. 2. $\quad$ Yu, C.; Huang, L., Cross-Linking Mass Spectrometry: An Emerging Technology for Interactomics and Structural Biology. Anal Chem 2018, 90 (1), 144-165.

3. Leitner, A.; Faini, M.; Stengel, F.; Aebersold, R., Crosslinking and Mass Spectrometry: An Integrated Technology to Understand the Structure and Function of Molecular Machines. Trends Biochem Sci 2016, 41 (1), 20-32.

4. O'Reilly, F. J.; Rappsilber, J., Cross-linking mass spectrometry: methods and applications in structural, molecular and systems biology. Nat Struct Mol Biol 2018, 25 (11), 1000-1008.

5. Chavez, J. D.; Bruce, J. E., Chemical cross-linking with mass spectrometry: a tool for systems structural biology. Curr Opin Chem Biol 2019, 48, 8-18.

6. Matzinger, M.; Mechtler, K., Cleavable Cross-Linkers and Mass Spectrometry for the Ultimate Task of Profiling Protein-Protein Interaction Networks in Vivo. J Proteome Res 2021, 20 (1), 78-93.

7. Klykov, O.; Steigenberger, B.; Pektas, S.; Fasci, D.; Heck, A. J. R.; Scheltema, R. A., Efficient and robust proteome-wide approaches for cross-linking mass spectrometry. Nat Protoc 2018, 13 (12), 2964-2990.

8. Kastritis, P. L.; O'Reilly, F. J.; Bock, T.; Li, Y.; Rogon, M. Z.; Buczak, K.; Romanov, N.; Betts, M. J.; Bui, K. H.; Hagen, W. J.; Hennrich, M. L.; Mackmull, M. T.; Rappsilber, J.; Russell, R. B.; Bork, P.; Beck, M.; Gavin, A. C., Capturing protein communities by structural proteomics in a thermophilic eukaryote. Mol Syst Biol 2017, 13 (7), 936.

9. $\quad$ Schweppe, D. K.; Chavez, J. D.; Lee, C. F.; Caudal, A.; Kruse, S. E.; Stuppard, R.; Marcinek, D. J.; Shadel, G. S.; Tian, R.; Bruce, J. E., Mitochondrial protein interactome elucidated by chemical cross-linking mass spectrometry. Proc Natl Acad Sci U S A 2017, 114 (7), 1732-1737.

10. Fasci, D.; van Ingen, H.; Scheltema, R. A.; Heck, A. J. R., Histone Interaction Landscapes Visualized by Crosslinking Mass Spectrometry in Intact Cell Nuclei. Mol Cell Proteomics 2018, 17 (10), 2018-2033.

11. Gotze, M.; lacobucci, C.; Ihling, C. H.; Sinz, A., A Simple Cross-Linking/Mass Spectrometry Workflow for Studying System-wide Protein Interactions. Anal Chem 2019, 91 (15), 10236-10244.

12. Steigenberger, B.; van den Toorn, H. W. P.; Bijl, E.; Greisch, J. F.; Rather, O.; Lubeck, M.; Pieters, R. J.; Heck, A. J. R.; Scheltema, R. A., Benefits of Collisional Cross Section Assisted Precursor Selection (caps-PASEF) for Cross-linking Mass Spectrometry. Mol Cell Proteomics 2020, 19 (10), 1677-1687. 
13. Chavez, J. D.; Lee, C. F.; Caudal, A.; Keller, A.; Tian, R.; Bruce, J. E., Chemical Crosslinking Mass Spectrometry Analysis of Protein Conformations and Supercomplexes in Heart Tissue. Cell Syst 2018, 6 (1), 136-141 e5.

14. Beveridge, R.; Stadlmann, J.; Penninger, J. M.; Mechtler, K., A synthetic peptide library for benchmarking crosslinking-mass spectrometry search engines for proteins and protein complexes. Nat Commun 2020, 11 (1), 742.

15. Erzberger, J. P.; Stengel, F.; Pellarin, R.; Zhang, S.; Schaefer, T.; Aylett, C. H. S.; Cimermancic, P.; Boehringer, D.; Sali, A.; Aebersold, R.; Ban, N., Molecular Architecture of the 40SelF1elF3 Translation Initiation Complex. Cell 2014, 159 (5), 12271228.

16. Fischer, L.; Rappsilber, J., Quirks of Error Estimation in Cross-Linking/Mass Spectrometry. Analytical Chemistry 2017, 89 (7), 3829-3833.

17. Liu, F.; Rijkers, D. T. S.; Post, H.; Heck, A. J. R., Proteome-wide profiling of protein assemblies by cross-linking mass spectrometry. Nature Methods 2015, 12 (12), 1179-+.

18. Walzthoeni, T.; Claassen, M.; Leitner, A.; Herzog, F.; Bohn, S.; Forster, F.; Beck, M.; Aebersold, R., False discovery rate estimation for cross-linked peptides identified by mass spectrometry. Nat Methods 2012, 9 (9), 901-3.

19. Fursch, J.; Kammer, K. M.; Kreft, S. G.; Beck, M.; Stengel, F., Proteome-Wide Structural Probing of Low-Abundant Protein Interactions by Cross-Linking Mass Spectrometry. Anal Chem 2020, 92 (5), 4016-4022.

20. Leitner, A.; Walzthoeni, T.; Aebersold, R., Lysine-specific chemical cross-linking of protein complexes and identification of cross-linking sites using LC-MS/MS and the xQuest/xProphet software pipeline. Nat Protoc 2014, 9 (1), 120-37.

21. Sailer, C.; Jansen, J.; Erzberger, J. P.; Stengel, F., A comprehensive landscape of 60 S ribosome biogenesis factors. bioRxiv 2021, 2021.05.11.443624.

22. Rinner, O.; Seebacher, J.; Walzthoeni, T.; Mueller, L. N.; Beck, M.; Schmidt, A.; Mueller, M.; Aebersold, R., Identification of cross-linked peptides from large sequence databases. Nat Methods 2008, 5 (4), 315-8.

23. Gotze, M.; Pettelkau, J.; Fritzsche, R.; Ihling, C. H.; Schafer, M.; Sinz, A., Automated assignment of MS/MS cleavable cross-links in protein 3D-structure analysis. J Am Soc Mass Spectrom 2015, 26 (1), 83-97.

24. Giese, S. H.; Fischer, L.; Rappsilber, J., A Study into the Collision-induced Dissociation (CID) Behavior of Cross-Linked Peptides. Mol Cell Proteomics 2016, 15 (3), 1094-104.

25. Yang, B.; Wu, Y. J.; Zhu, M.; Fan, S. B.; Lin, J.; Zhang, K.; Li, S.; Chi, H.; Li, Y. X.; Chen, H. F.; Luo, S. K.; Ding, Y. H.; Wang, L. H.; Hao, Z.; Xiu, L. Y.; Chen, S.; Ye, K.; He, S. M.; Dong, M. Q., Identification of cross-linked peptides from complex samples. Nat Methods 2012, 9 (9), 904-6. 
26. Chen, Z. L.; Meng, J. M.; Cao, Y.; Yin, J. L.; Fang, R. Q.; Fan, S. B.; Liu, C.; Zeng, W. F.; Ding, Y. H.; Tan, D.; Wu, L.; Zhou, W. J.; Chi, H.; Sun, R. X.; Dong, M. Q.; He, S. M., A high-speed search engine pLink 2 with systematic evaluation for proteome-scale identification of cross-linked peptides. Nat Commun 2019, 10 (1), 3404. 27. Veit, J.; Sachsenberg, T.; Chernev, A.; Aicheler, F.; Urlaub, H.; Kohlbacher, O., LFQProfiler and RNP(xl): Open-Source Tools for Label-Free Quantification and ProteinRNA Cross-Linking Integrated into Proteome Discoverer. J Proteome Res 2016, 15 (9), 3441-8.

28. Eisele, M. R.; Reed, R. G.; Rudack, T.; Schweitzer, A.; Beck, F.; Nagy, I.; Pfeifer, G.; Plitzko, J. M.; Baumeister, W.; Tomko, R. J., Jr.; Sakata, E., Expanded Coverage of the 26S Proteasome Conformational Landscape Reveals Mechanisms of Peptidase Gating. Cell Rep 2018, 24 (5), 1301-1315 e5.

29. Combe, C. W.; Fischer, L.; Rappsilber, J., xiNET: cross-link network maps with residue resolution. Mol Cell Proteomics 2015, 14 (4), 1137-47.

30. Perez-Riverol, Y.; Bai, J.; Bandla, C.; García-Seisdedos, D.; Hewapathirana, S.; Kamatchinathan, S.; Kundu, D. J.; Prakash, A.; Frericks-Zipper, A.; Eisenacher, M.; Walzer, M.; Wang, S.; Brazma, A.; Vizcaíno, J. A., The PRIDE database resources in 2022: a hub for mass spectrometry-based proteomics evidences. Nucleic Acids Res 2022, 50 (D1), D543-d552. 


\section{Supplemental Figures}

bioRxiv preprint doi: https://doi.org/10.1101/2022.02.03.478943; this version posted February 3, 2022. The copyright holder for this preprint (which was not certified by peer review) is the author/funder, who has granted bioRxiv a license to display the preprint in perpetuity. It is made available under aCC-BY-NC-ND 4.0 International license.

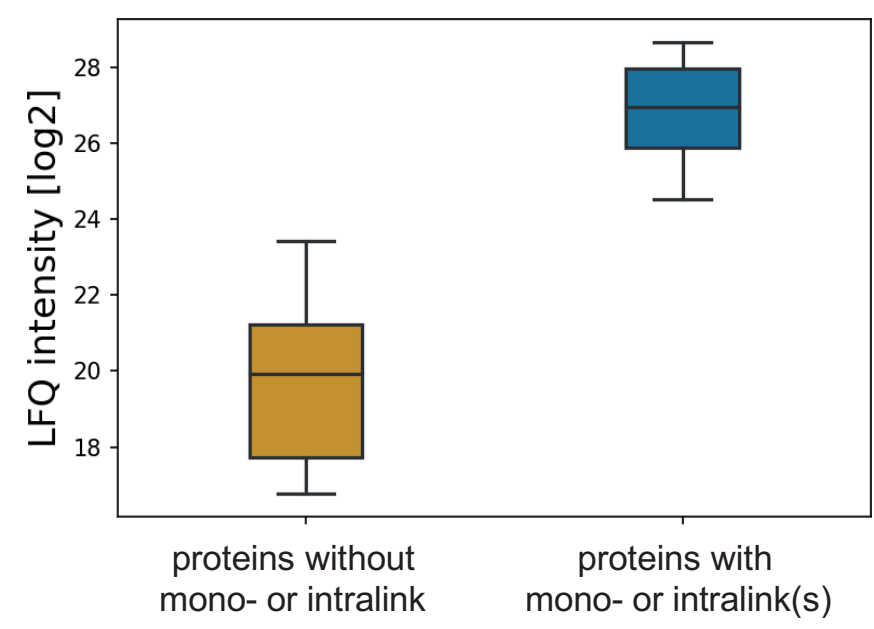

Supplementary Figure 1: Relative Abundance of proteins without mono- or intra-protein link that were filtered-out by the mi-filter (yellow) compared to the relative abundance of proteins with a mono- or intra-protein link (blue). LFQ intensities were calculated based on peptides which were not modified by the crosslinking reagent DSS. 
bioRxiv preprint doi: https://doi.org/10.1101/2022.02.03.478943; this version posted February 3, 2022. The copyright holder for this preprint (which was not certified by peer review) is the author/funder, who has granted bioRxiv a license to display the preprint in perpetuity. It is made

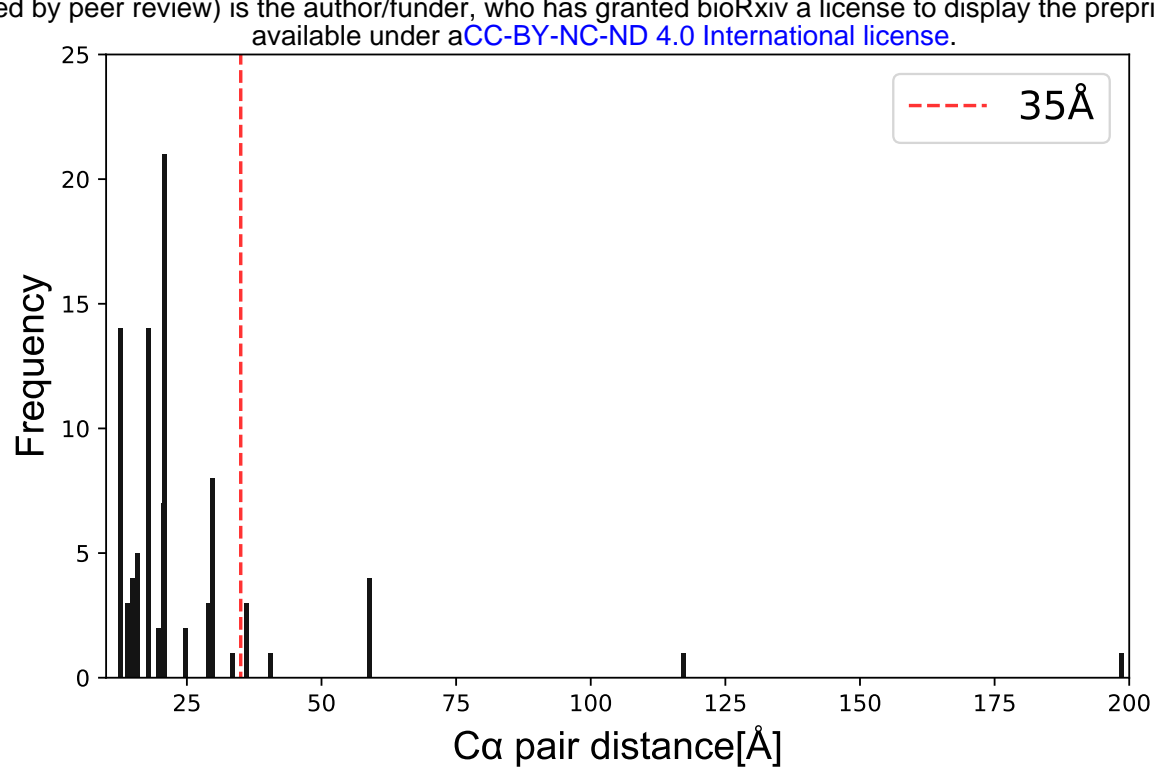

Supplementary Figure 2: Histogram of mapped distances of all cross-linked residues within the mi-filtered $26 \mathrm{~S}$ proteasome data set versus their frequency. The $35 \mathrm{~A}^{\circ}$ threshold is indicated as a red dotted line. 